\title{
Expression of TRAF6 and ubiquitin mRNA in skeletal muscle of gastric cancer patients
}

\author{
Yuan-Shui Sun*, Zai-Yuan Ye*, Zhen-Yuan Qian, Xiao-Dong Xu and Jun-Feng Hu
}

\begin{abstract}
Objective: To investigate the prognostic significance of tumor necrosis factor receptor (TNFR),-associated factor 6 (TRAF6),-and ubiquitin in gastric cancer patients.

Methods: Biopsies of the rectus abdominis muscle were obtained intra operatively from 102 gastric cancer patients and 29 subjects undergoing surgery for benign abdominal diseases, and muscle TRAF6 and ubiquitin mRNA expression and proteasome proteolytic activities were assessed.

Results: TRAF6 was significantly upregulated in muscle of gastric cancer compared with the control muscles. TRAF6 was upregulated in 67.65\% (69/102) muscle of gastric cancer. Over expression of TRAF6 in muscles of gastric cancer were associated with TNM stage, level of serum albumin and percent of weight loss. Ubiquitin was significantly upregulated in muscle of gastric cancer compared with the control muscles. Ubiquitin was upregulated in 58.82\% (60/102) muscles of gastric cancer. Over expression of ubiquitin in muscles of gastric cancer were associated with TNM (Tumor-Node-Metastasis) stage and weight loss. There was significant relation between TRAF6 and ubiquitin expression.

Conclusions: We found a positive correlation between TRAF6 and ubiquitin expression, suggesting that TRAF6 may up regulates ubiquitin activity in cancer cachexia. While more investigations are required to understand its mechanisms of TRAF6 and ubiquitin in skeletal muscle. Correct the catabolic-anabolic imbalance is essential for the effective treatment of cancer cachexia.
\end{abstract}

Keyword: Gastric cancer, TRAF6, Ubiquitin, Mrna, Skeletal muscle, Cachexia

\section{Introduction}

Cancer cachexia is a complex metabolic condition characterized by loss of skeletal muscle. Common clinical manifestations include muscle wasting, anemia, reduced caloric intake, and altered immune function, which contribute to increased disability, fatigue, diminished quality of life, and reduced survival [1-3]. Many patients with cancer present with weight loss at diagnosis, and much of this weight loss can be attributed to muscle wasting. Cancer cachexia has been viewed as an end-of-life condition in patients with advanced or incurable malignancies that was managed primarily through palliative approaches. However, cachexia and associated skeletal muscle loss may be present early in the progression of cancer, indicating the importance of earlier diagnosis and treatment.

\footnotetext{
*Correspondence: sunshui818@126.com; zaiyuanye@163.com Departments of Gastrointestinal Surgery, Zhejiang Provincial People's Hospital, Hangzhou 310014, PR China
}

The prevalence of cancer cachexia varies depending on the type of malignancy, with the greatest frequency of weight loss (50\%-85\% of patients) observed in gastrointestinal, pancreatic, lung, and colorectal cancers at diagnosis and before initiation of chemotherapy [4]. One common mechanism associated with skeletal muscle protein degradation in cancer cachexia is the activation of the adenosine triphosphate-dependent ubiquitin-proteasome proteolytic path way $[5,6]$. This system plays a major role in muscle wasting and, more specifically, in the breakdown of myofibrillar proteins. Certainly, the mechanisms of muscle wasting in cancer cachexia are complex. They involve multiple host and tumor factors, decreased levels of testosterone and insulin-like growth factor-1 (IGF-1), and decreased food intake, contributing to both antianabolic and procatabolic processes $[7,8]$. The study demonstrate that the expression level of tumor necrosis factor $(\alpha)$ receptor adaptor protein 6 (TRAF6), a protein involved in 
Table 1 Summary of characteristics of gastric cancer patients and control

\begin{tabular}{lcccc}
\hline & Controls $(\mathbf{n}=\mathbf{2 9})$ & Gastric cancer $(\mathbf{n}=\mathbf{1 0 2})$ & $\mathbf{t} / \boldsymbol{X}^{\mathbf{2}}$ & $\mathbf{P}$ Value \\
\hline Age, $\mathrm{y}$ & $61.88 \pm 6.49$ & $62.13 \pm 6.54$ & 0.053 & 0.959 \\
Sex (M:F) & $21: 8$ & $72: 30$ & 0.037 & 0.848 \\
Weight loss & $65.50 \pm 4.84$ & $57.38 \pm 6.28$ & 2.899 & 0.012 \\
BMI & $24.13 \pm 1.81$ & $21.00 \pm 1.31$ & 3.96 & 0.001 \\
Serum albumin, g/L & $41.38 \pm 6.09$ & $33.75 \pm 3.11$ & 3.15 & 0.007 \\
PNI & $45.25 \pm 3.62$ & $37.18 \pm 3.74$ & 5.26 & 0.0001 \\
\hline
\end{tabular}

receptor-mediated activation of several signaling pathways, is enhanced in skeletal muscle during atrophy $[9,10]$.

Here, we analyzed the prognostic significance of tumor necrosis factor receptor (TNFR)-associated factor 6 (TRAF6), and ubiquitin in gastric cancer patients. The current study were to examine the expression of TRAF6 and ubiquitin in skeletal muscle specimens of patients with gastric cancer, to explore the possible correlation among TRAF6, ubiquitin mRNA expression and cachexia.

\section{Methods}

Patients and tissue samples

Skeletal muscle tissues were collected from one hundred and two patients with gastric cancer (median age 61.0y, range 42-88y; 24 male, 10 female) from the Department of Surgery, Zhejiang Provincial People's Hospital from January 2008 to January 2011. Patients' characteristics are showed in Table 1. Diagnosis of gastric cancer was performed by endoscopic biopsy. Twenty-nine patients undergoing surgery for benign abdominal diseases served as a control group, there were 12 cholelithiasis, 9 inguinal hernia, 8 hemangioma of liver. Gastric cancer patients and controls were similar in terms of age and sex distribution. Nevertheless, gastric cancer patients showed a significantly lower body mass index, serum albumin levels and prognostic nutritional index. Exclusion criteria for both groups were considered: acute or chronic renal failure, liver failure, diabetes, metabolic acidosis, sepsis, AIDS, inflammatory bowel disease, autoimmune disorders, chronic heart failure, and hyperthyroidism. The study was approved by our hospital ethics committees. Written informed consent for the study procedures was obtained from the patients.

\section{Nutritional assessment}

The nutritional assessment included anthropometric [height, actual body weight, \%WL, body mass index (BMI), usual body weight], immunological (total lymphocyte count), and biochemical (serum albumin) indexes. Routine blood test was determined using completely automatic blood cell count analyzer (Beckman-Coulter -MAXM, American). Liver function was determined using Completely automatic biochemistry analyzer (Beckman-Coulter SYNCHRON LX 20,
American) (Table 1). The PNI(prognostic nutritional index) was calculated as follows: $\mathrm{PNI}=10 \times$ serum albu$\min (\mathrm{g} / 100 \mathrm{ml})+0.005 \times$ total lymphocyte count $/ \mathrm{mm}^{3}$ of peripheral blood [11].

\section{Muscle biopsy}

A biopsy specimen was obtained from the rectus abdominis muscle during the initial phase of the operation. The anterior sheet of the rectus abdominis muscle was opened with scissors after skin incision and dissection through the subcutaneous fat, and a muscle biopsy specimen weighing about $1.0 \mathrm{~g}$ was obtained. The biopsy specimen was divided into two portions that were immediately frozen in liquid nitrogen and then stored at $-80^{\circ} \mathrm{C}$ until analysis. No complications occurred from the biopsy procedure.

\section{Real-time quantitative RT-PCR}

Total RNA from rectus abdominis muscle was extracted by TRIzol reagent and cDNAs were reverse-transcribed by Revert Aid TM reverse transcriptase. Real-time PCR was carried out using the ABI PRISM 7700 Sequence Detection System (Applied Bio systems) at $50^{\circ} \mathrm{C}$ for $2 \mathrm{~min}, 95^{\circ} \mathrm{C}$ for $10 \mathrm{~min}$, followed by 50 cycles at $95^{\circ} \mathrm{C}$ for $15 \mathrm{~s}$, and at $60^{\circ} \mathrm{C}$ for $1 \mathrm{~min}$. The primers for GAPDH (224 bp) were $5^{\prime}-$ TGAAGGTCGGAGTCAACGG-3' (sense) and 5'- CTGG AAGATGGTGATGGGATT-3' (antisense). The primers for TRAF6 (134 bp) were 5'-GCCTGGGTGACAGAGTG C-3' (sense) and 5'-AATGACTACTTATGGCTCCTTT

Table 2 The expression of TRAF6 in muscle of cancer patients

\begin{tabular}{lllll}
\hline & low & high & $\boldsymbol{X}^{\mathbf{2}}$ & P Value \\
\hline Percent weight loss & & & 12.9 & 0.001 \\
$\geq 10$ & 9 & 45 & & \\
$<10$ & 24 & 24 & & \\
Serum albumin,g/L & & & 20.05 & 0.001 \\
$\geq 35$ & 24 & 18 & & \\
$<35$ & 9 & 51 & & \\
TNM stage & & & 13.33 & 0.001 \\
I-II & 21 & 18 & & \\
III-IV & 12 & 51 & & \\
\hline
\end{tabular}




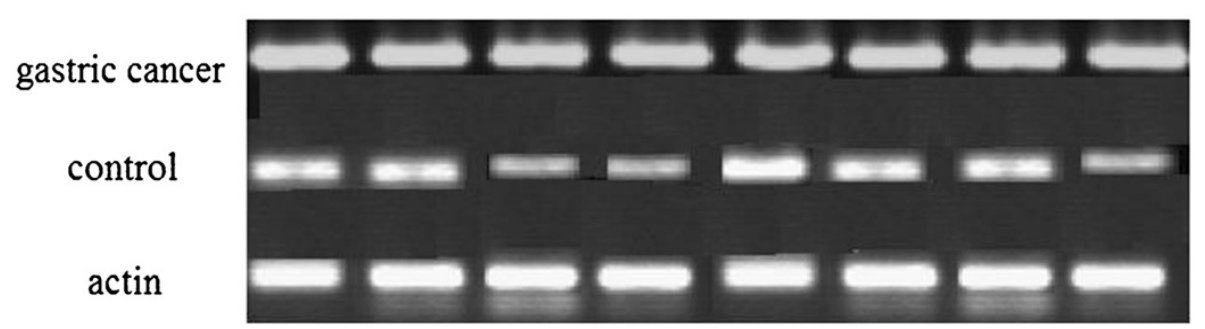

Figure 1 The level of TRAF6 protein in muscle of cancer patients and control.

TC-3' (antisense). The primers for ubiquitin(165 bp) were 5'-CCCTGGATGTGATGGTGTC-3' (sense) and 5'-CTCG TTGTCCCTGTTGCTG-3' (antisense). The expression of GAPDH was used to normalize that of the target genes. Each assay was done in triplicate, the average was calculate, and the expression level of TRAF6 and ubiquitin was expressed as $2^{-\Delta \Delta \mathrm{Ct}}, \Delta \mathrm{Ct}=\mathrm{Ct}$ (Target) $-\mathrm{Ct}(\mathrm{GAPDH})$.

\section{Immunoblotting}

Cells were lysed in RIPA buffer $(150 \mathrm{mM} \mathrm{NaCl}$, $10 \mathrm{mM}$ Tris, $\mathrm{pH} 7.5,1 \%$ NP40, $1 \%$ deoxycholate, $0.1 \%$ SDS, protease inhibitor cocktail (Roche)). Total proteins were fractionated using the NuPAGE $4-12 \%$ BisTris gradient gel (Invitrogen) and transferred onto PVDF membrane. Membranes were blocked with 5\% non-fat milk in PBS/Tween-20, and incubated with antibodies against TRAF6 (Santa Cruz), ubiquitin (Santa Cruz), and $\beta$-actin (Abcam).

\section{Statistical analysis}

In order to analyze the relationship among the expression of TRAF6 and ubiquitin and nutritional status of patients (percent weight loss, serum albumin), according to the literature [12], they were divided into two groups (percent weight loss $\geq 10$ and $<10$, serum albumin $\geq 35$ and $<35$ ). All statistical analyses were performed using SPSS16.0 software. Measurement data were analyzed using the Student's $t$ test, while categorical data were studied using $X^{2}$ or Fisher exact tests. Statistical significance was set at $\mathrm{P}<0.05$.

\section{Results}

The expression of TRAF6 in muscle of control and cancer patients

Tumor necrosis factor $(\alpha)$ receptor adaptor protein 6 (TRAF6), a protein involved in receptor-mediated activation of several signaling pathways, is enhanced in skeletal muscle during atrophy. We assessed the expression of TRAF6 in 29 control muscles and 102 patient muscles. TRAF6 was significantly upregulated in muscle of gastric cancer compared with the control muscles $(\mathrm{P}<0.05)$. TRAF6 was upregulated in $67.65 \%(69 / 102)$ muscle of gastric cancer. Overexpression of TRAF6 in muscles of gastric cancer were associated with TNM stage, level of serum albumin and percent of weight loss $(\mathrm{P}>0.05)$ (Table 2). We also analyze the expression of TRAF6 in 8 muscles of control and cancer patients by western blotting, the results show the expression of TRAF6 in muscle of cancer patients were higher than control (Figure 1).

\section{The expression of ubiquitin in muscle of control and} cancer patients

We assessed the expression of ubiquitin in 29 control muscles and 102 patient muscles. Ubiquitin was significantly upregulated in muscle of gastric cancer compared with the control muscles $(\mathrm{P}<0.05)$. Ubiquitin was upregulated in 58.82\% (60/102) muscles of gastric cancer. Over expression of ubiquitin in muscles of gastric cancer were associated with TNM stage and weight loss $(\mathrm{P}>0.05)$ (Table 3). In order to analyze the expression of ubiquitin protein with quantitation, 8 muscle of control and cancer patients were detectec by western blotting, the study indicated the expression of ubiquitin in 5 muscle of cancer patients were higher than control (Figure 2).

\section{Association between expression of TRAF6 and ubiquitin}

Seventeen cases of gastric cancer had high expression of both TRAF6 and ubiquitin, and eight cases of gastric cancer had low expression of both TRAF6 and ubiquitin.

Table 3 The expression of ubiquitin in muscle of cancer patients

\begin{tabular}{lllll}
\hline & low & high & $\boldsymbol{X}^{\mathbf{2}}$ & P Value \\
\hline Percent weight loss & & & 11.78 & 0.001 \\
$\geq 10$ & 15 & 42 & & \\
$<10$ & 27 & 18 & & \\
Serum albumin,g/L & & & 15.74 & 0.001 \\
$\geq 35$ & 27 & 15 & & \\
$<35$ & 15 & 45 & & \\
TNM stage & & & 20.52 & 0.001 \\
I-II & 27 & 12 & & \\
III-IV & 15 & 48 & & \\
\hline
\end{tabular}




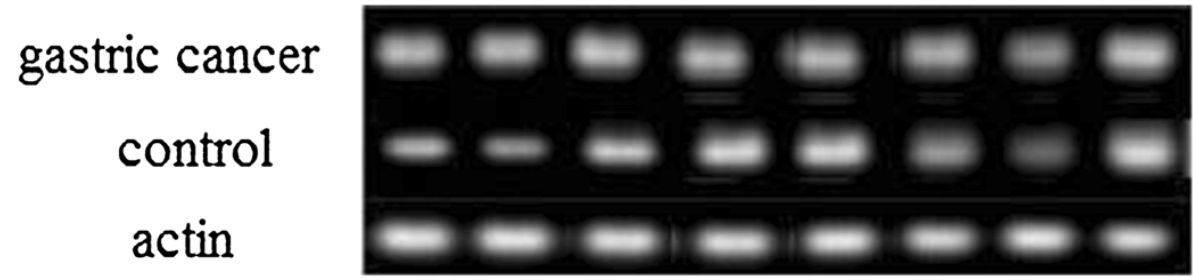

Figure 2 The level of ubiquitin protein in muscle of cancer patients and control.

There was significant between TRAF6 and ubiquitin expression $\left(\chi^{2}=6.68 ; P=0.01\right)$ (Table 4 , Figure 3$)$.

\section{Discussion}

In healthy individuals, skeletal muscle metabolism requires a balance of anabolic and catabolic processes, resulting in a continuous renewal of muscle proteins without a net change in overall muscle mass. However, in cancer cachexia and other chronic illnesses, the muscle wasting were associated with the reduced rate of protein synthesis, increased protein degradation, or a combination of both contributes [13]. One common mechanism associated with skeletal muscle protein degradation in cancer cachexia is the activation of the adenosine triphosphate-dependent ubiquitin-proteasome proteolytic path way, this system plays a major role in muscle wasting $[5,6]$. The study showed muscle ubiquitin mRNA was hyper expressed in gastric cancer patients compared to controls [14], the ubiquitinproteasome proteolytic system play important role in the pathogenesis of muscle protein hyper catabolism in cancer cachexia. To investigate the role of ubiquitin expression in the skeletal muscle of gastric cancer patients. We assessed the expression of ubiquitin in 29 control muscles and 102 patient muscles. Ubiquitin was significantly upregulated in muscle of gastric cancer compared with the control muscles. Over expression of ubiquitin in muscle of gastric cancer were associated with TNM stage and weight loss.

Skeletal muscle wasting is a major reason for morbidity and mortality in many chronic disease states, disuse conditions and aging. The ubiquitin-proteasome and autophagy-lysosomal systems are the two major proteolytic pathways involved in regulation of both physiological and pathological muscle wasting. The study

Table 4 Association between expression of TRAF6 and ubiquitin

\begin{tabular}{lcccc}
\hline \multirow{2}{*}{$\begin{array}{c}\text { Clinical } \\
\text { parameters }\end{array}$} & \multicolumn{4}{c}{ TRAF6 } \\
\cline { 2 - 5 } & high & low & $\mathbf{X}^{\mathbf{2}}$ & $\mathbf{P}$ \\
\hline ubiquitin & & & 20.05 & 0.001 \\
high & $51(85.0 \%)$ & $9(15.0 \%)$ & & \\
low & $18(42.9 \%)$ & $24(57.1 \%)$ & & \\
\hline
\end{tabular}

demonstrate that the expression level of tumor necrosis factor $(\alpha)$ receptor adaptor protein 6 (TRAF6), a protein involved in receptor-mediated activation of several signaling pathways, is enhanced in skeletal muscle during atrophy $[9,10]$. To explore the relation of TRAF6 expression in the skeletal muscle of gastric cancer patients. We assessed the expression of TRAF6 in 29 control muscles and 102 patient muscles. TRAF6 was significantly upregulated in muscle of gastric cancer compared with the control muscles, Overexpression of TRAF6 in muscle of gastric cancer were associated with TNM stage, the level of serum albumin and percent of weight loss. The study showed overexpression of TRAF6 may play important role in gastric cancer cachexia.

Paul's study discover that TRAF6 possesses E3 ubiquitin ligase activity causing lysine-63-linked polyubiquitination of target proteins. Muscle-wasting stimuli could up regulate the expression of TRAF6 and auto-ubiquitination. Muscle-specific depletion of TRAF6 preserves skeletal muscle mass in a mouse model of cancer cachexia or denervation. Inhibition of TRAF6 also blocks the expression of the components of the ubiquitinproteasome system (UPS) and auto phagosome formation in atrophying skeletal muscle [15]. We also examined TRAF6 expression in skeletal muscle with gastric

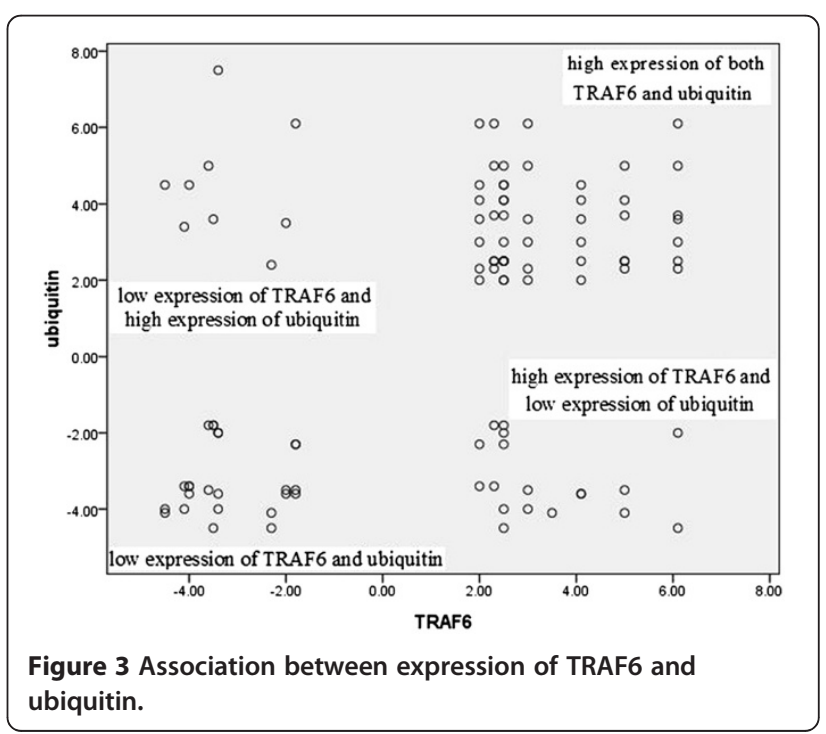


cancer and its correlation with ubiquitin status. We found a positive correlation between TRAF6 and ubiquitin expression, suggesting that TRAF6 may up regulates ubiquitin activity in cancer cachexia. While more investigations are required to understand its mechanisms of TRAF6 and ubiquitin in skeletal muscle. Correct the catabolic-anabolic imbalance is essential for the effective treatment of cancer cachexia.

\section{Competing interests}

The authors declared that they have no competing interest.

\section{Authors' contributions}

Y-SS and Z-YY design the study, Z-YQ, X-DX, and J-FH carried out the Realtime quantitative RT-PCR and Immunoblotting, $Y$-SS drafted the manuscript. All authors read and approved the final manuscript.

\section{Acknowledgments}

Work was supported by Zhejiang Provincial Department of Science and Technology Research Foundation (2011C33009).

Received: 18 August 2012 Accepted: 24 September 2012

Published: 26 September 2012

\section{References}

1. Gullett N, Rossi P, Kucuk O, Johnstone PA: Cancer-induced cachexia: a guide for the oncologist. J Soc Integr Oncol 2009, 7(4):155-169.

2. Evans WJ: Skeletal muscle loss: cachexia, sarcopenia, and inactivity. Am J Clin Nutr 2010, 91(4):1123S-1127S

3. Evans WJ, Morley JE, Argilés J, et al: Cachexia: a new definition. Clin Nutr 2008, 27(6):793-799.

4. Dodson S, Baracos VE, Jatoi A, et al: Muscle wasting in cancer cachexia: clinical implications, diagnosis, and emerging treatment strategies. Annu Rev Med 2011, 62:265-279.

5. Baracos VE: Cancer-associated cachexia and underlying biological mechanisms. Annu Rev Nutr 2006, 26:435-461.

6. Tisdale MJ: Mechanisms of cancer cachexia. Physiol Rev 2009, 89(2):381-410.

7. Chopard A, Hillock S, Jasmin BJ: Molecular events and signalling pathways involved in skeletal muscle disuse-induced atrophy and the impact of counter measures. J Cell Mol Med 2009, 13(9B):3032-3050.

8. Kubrak C, Olson K, Jha N, et al: Nutrition impact symptoms: key determinants of reduced dietary intake, weight loss, and reduced functional capacity of patients with head and neck cancer before treatment. Head Neck 2010, 32(3):290-300.

9. Paul PK, Gupta SK, Bhatnagar S, et al: Targeted ablation of TRAF6 inhibits skeletal muscle wasting in mice. J Cell Biol 2010, 191(7):1395-1411.

10. Kumar A, Bhatnagar S, Paul PK: TWEAK and TRAF6 regulate skeletal muscle atrophy. Curr Opin Clin Nutr Metab Care 2012, 15(3):233-239.

11. Onodera T, Goseki N, Kosaki G: Prognostic nutritional index in gastrointestinal surgery of malnourished cancer patients. Nippon Geka Gakkai Zasshi 1984, 85:1001.

12. Bossola M, Muscaritoli M, Costelli P, Grieco G, Bonelli G, Pacelli F, Rossi Fanelli F, Doglietto GB, Baccino FM: Increased muscle proteasome activity correlates with disease severity in gastric cancer patients. Ann Surg 2003, 237(3):384-389.

13. Baracos VE: Management of muscle wasting in cancer-associated cachexia: understanding gained from experimental studies. Cancer 2001 92(6 Suppl):1669-1677.

14. Bossola M, Muscaritoli M, Costelli $P$, et al: Increased muscle proteasome activity correlates with disease severity in gastric cancer patients. Ann Surg 2003, 237(3):384-389.
15. Paul PK, Kumar A: TRAF6 coordinates the activation of autophagy and ubiquitin-proteasome systems in atrophying skeletal muscle. Autophagy 2011, 7(5):555-556

doi:10.1186/1756-9966-31-81

Cite this article as: Sun et al:: Expression of TRAF6 and ubiquitin mRNA in skeletal muscle of gastric cancer patients. Journal of Experimental \& Clinical Cancer Research 2012 31:81.

\section{Submit your next manuscript to BioMed Central and take full advantage of:}

- Convenient online submission

- Thorough peer review

- No space constraints or color figure charges

- Immediate publication on acceptance

- Inclusion in PubMed, CAS, Scopus and Google Scholar

- Research which is freely available for redistribution 